\title{
Uso de imagens de satélite na discriminação de solos desenvolvidos de basalto e arenito na região de Paraguaçu Paulista
}

\author{
José Alexandre M. Demattê(1), Dolorice Moreti(1), Ana Carolina Feitosa de Vasconcelos ${ }^{(1)}$ e Aline Marques Genú(1) \\ (1)Universidade de São Paulo (USP), Escola Superior de Agricultura "Luiz de Queiroz" (Esalq), Dep. de Solos e Nutrição de Plantas, Av. Pádua \\ Dias, 11, Caixa Postal 9, CEP 13418-900, Piracicaba, SP. E-mail: jamdemat@carpa.ciagri.usp.br, dmoreti@esalq.usp.br, acfvasco@esalq.usp.br, \\ amgenu@esalq.usp.br
}

\begin{abstract}
Resumo - O objetivo deste trabalho foi avaliar o potencial de discriminação de solos, mediante informações espectrais obtidas por sensor remoto em satélite. Os solos apresentaram variações na forma da curva espectral e intensidades de reflectância. Os Nitossolos apresentaram baixa intensidade de reflectância e os Neossolos, bastante alta. Os Latossolos Vermelhos, com diferentes texturas, apresentaram aumento na intensidade de reflectância, na sequiência dos mais argilosos para os mais arenosos. Solos eutróficos apresentaram maiores intensidades de reflectância que os álicos. A análise discriminante indicou um erro geral de $0,028 \%$. As amostras confundidas geralmente ocorreram entre os solos com características semelhantes. A metodologia empregada na coleta de dados espectrais de satélites mostrou-se eficiente, tornando possível discriminar os solos da região estudada e, assim, agilizar a confecção de mapas de solos.
\end{abstract}

Temos para indexação: imagens orbitais, reflectância, mapa de solos, fertilidade do solo.

\section{Satellite images on the discrimination of soils developed from basalt and sandstone of Paraguaçu Paulista region}

\begin{abstract}
The objective of this work was to evaluate soil characterization and discrimination by spectral information obtained from satellite sensor. Soils presented variation on the spectral signature, and on their spectral intensity. Typic Argiudoll (Alfisols) presented low spectral intensity, and Typic Quartzpisament presented a high spectral intensity. Typic Haplorthox (Oxisols) presented increments in its reflectance according to the texture. Eutrophic soils presented greater reflectance intensities than Alic soils. The discriminate analyses indicate a $0.028 \%$ error. Mistake samples occurred due to similar characteristics of soils. The methodology used to collect spectral data from satellite sensor showed to be efficient, and made possible soil discrimination, as well as faster soil survey of the presented region.
\end{abstract}

Index terms: orbital images, reflectance, soil survey, soil fertility.

\section{Introdução}

O Brasil é o segundo maior produtor agrícola mundial (IBGE, 2003a), com previsão de 119,72 milhões de toneladas de grãos em 2003, contra 97,174 milhões de toneladas em 2002 (IBGE, 2003b) e, em produção global, está em posição imediatamente abaixo dos Estados Unidos da América do Norte. Tal condição vem sendo alcançada, dentre inúmeros fatores, em conseqüência do uso de tecnologia, que visa aumentos na produtividade e diminuição dos custos, e sustentabilidade da produção.
O desenvolvimento de tecnologia baseia-se nos estudos do solo, da planta e no clima. Desses, destaca-se o solo, substrato onde as plantas se desenvolvem, com sua diversidade, influenciada pelo material de origem e condições climáticas. O reconhecimento, a caracterização e o estudo do solo são de grande importância no mapeamento e uso racional da terra. As informações do meio físico, em escalas adequadas, ainda são raras no Brasil. Com relação aos levantamentos de solos, Oliveira (2001) observa que apenas 5\% dos solos brasileiros foram mapeados na escala 1:25.000, $15 \%$ a $20 \%$ na escala de 1:100.000 e 35\% em escala de 1:250.000. 
O Estado de São Paulo possui mapas mais detalhados e abrangentes, com 22 quadrículas mapeadas em escala de 1:100.000.

Os mapas de solos não são utilizados nos planejamentos agrícolas devido a falta de recursos para sua elaboração. Assim, novos mapas precisam ser desenvolvidos e outros melhorados. O custo, porém, é alto, e há necessidade de meios que auxiliem nessa tarefa. O sensoriamento remoto tem sido considerado uma importante ferramenta, nos estudos de solos (Nanni, 2000), por permitir a caracterização espectral.

Sensoriamento Remoto é a ciência e a arte de obtenção de informações de uma área ou fenômeno, pela análise de dados por um sensor, que não está em contato com o objeto, com a área ou com o fenômeno a ser investigado (Lillesand \& Kiefer, 2000). O sensoriamento remoto estuda a interação entre as características do objeto e a energia eletromagnética por ele refletida, permitindo detectar informações qualitativas e quantitativas do solo. A reflectância do solo é uma propriedade influenciada pela combinação heterogênea de matéria mineral, orgânica, ar e água que compõem os solos. Portanto, os parâmetros de solo como matéria orgânica, material de origem, mineralogia, óxidos de ferro, umidade, textura e estrutura interferem diretamente na intensidade de reflectância do solo (Ben-Dor, 2002).

As curvas de reflectância espectral de alvos apresentam formas diversas que são identificadas pela maior ou menor absorção da luz pelo alvo em estudo, em várias faixas de comprimento de ondas. Existe um número razoável de trabalhos que utilizam sensores terrestres para o estudo do comportamento espectral de solos tropicais (Demattê \& Garcia, 1999; Vitorello \& Galvão, 1999; Demattê et al., 2000a).

Os estudos iniciaram-se com aerofotos e uso de dados dos sensores multiespectrais MSS, TM-LANDSAT e HRV-SPOT. Os trabalhos desenvolvidos comprovam a potencialidade da utilização de dados de sensores, nas pesquisas de caracterização dos solos a serem aplicadas no seu levantamento e mapeamento. $\mathrm{O}$ avanço na tecnologia de sensores remotos e sistemas computacionais permitiu a integração entre a análise de imagens orbitais e sistemas de informações geográficas, e facilitou o levantamento de solos (McBratney et al., 2003).

Análises das propriedades do solo, associadas às curvas de intensidade de reflectância, vêm sendo utilizadas principalmente no nível de espectroradiometria em laboratório, criando bibliotecas espectrais. Tais estudos validaram a informação espectral em nível terrestre (Epiphanio et al., 1992; Shepherd \& Walsh, 2002). Torna-se necessário, porém, saber como se comportam os dados dos solos tropicais, no nível orbital, e se podem ser discriminados com maior detalhamento, levando-se em consideração atributos físicos (textura) e químicos (fertilidade) de tais solos.

O objetivo deste trabalho foi avaliar o potencial de discriminação de solos, por meio de informações espectrais obtidas pelo sensoriamento remoto por satélite.

\section{Material e Métodos}

A área localiza-se no sudoeste do Estado de São Paulo e apresenta como Municípios principais Paraguaçú Paulista, Gardênia e Iepê. O clima é mesotérmico, a temperatura média anual oscila em torno de $22^{\circ} \mathrm{C}$, com precipitação média anual na faixa de 1.400 a $1.600 \mathrm{~mm}$. Delimitada pelas coordenadas geográficas, $22^{\circ} 24^{\prime} 53^{\prime \prime S}$ e $50^{\circ} 34$ '35"W, a região apresenta um relevo suavemente ondulado, com altitudes que variam entre $200 \mathrm{~m}$ e $400 \mathrm{~m}$.

Há predomínio de material sedimentar recente sobre rochas do arenito Bauru, de acordo com as cartas geológicas (Instituto de Geociência e Ciências Exatas, 1982). Na direção de Gardênia a Iepê, há ocorrência de rochas basálticas da formação Serra Geral; essas rochas eruptivas básicas ocorrem, principalmente, nos vales dos principais rios como o Paranapanema.

O mapeamento dos solos foi realizado em nível semidetalhado, de acordo com Embrapa (1996), utilizando-se como mapas bases a carta planialtimétrica escala 1:25.000 e imagens de satélite.

Foram alocados e georreferenciados 294 pontos de tradagem, para coleta de amostras de terra, representando uma área de 25.000 ha. Posteriormente, as amostras foram coletadas com trado, nas profundidades de 0-20, 40-60 e 80-100 cm. Foram demarcados 20 perfis representativos das classes de solos detectadas e, com base na descrição morfológica (Lemos \& Santos, 1996), a nomenclatura foi estabelecida, conforme Embrapa (1999).

Com o objetivo de avaliar a variação do componente espectral entre as classes de solos, foram utilizadas as bandas 1, 2, 3, 4, 5 e 7 do satélite Landsat-7, sensor ETM+ (Enhanced Thematic Mapper), referente a 19 de outubro de 2000.

Utilizando-se uma composição colorida 5R4G3B (falsa cor) impressa, foram desenhadas linhas preliminares 
de separação das unidades de mapeamento de solo, com base nos conceitos fundamentais da fotopedologia e interpretação de imagens, como aspecto da rede de drenagem, tonalidade, uso da terra, vegetação, toposseqüências e litologia.

Em seguida, com base na interpretação dos dados analíticos de campo e da imagem de satélite, foram ajustados os limites das unidades de mapeamento, e transformados para o formato vetorial (digitalizado) no software SPRING (INPE, 1999a) que, juntamente com os talhões, estradas e composições coloridas geraram o mapa de solos em nível semidetalhado.

Para a análise granulométrica usou-se o densímetro, com hexametafosfato de sódio como dispersante (Camargo et al., 1987). Foram determinados o pH $\left(\mathrm{CaCl}_{2}\right)$, matéria orgânica, fósforo pelo método da resina, potássio, cálcio, magnésio e alumínio. A capacidade de troca de cátions (CTC), a saturação por bases e por alumínio foram calculadas, conforme Raij et al. (1987).

Para uma melhor avaliação do comportamento espectral dos solos, os mesmos foram classificados quanto ao teor de argila do horizonte B em: 1) média-arenosa: $150-250 \mathrm{~g} \mathrm{~kg}^{-1}$ de argila; 2) média-argilosa: 250$350 \mathrm{~g} \mathrm{~kg}^{-1} \mathrm{e}$ 3) argilosa: $>350 \mathrm{~g} \mathrm{~kg}^{-1} \mathrm{e}$, quanto à fertilidade em: eutróficos (e): $\mathrm{V} \geq 50 \%$; distróficos (d): $\mathrm{V}<50 \%$ e álicos (a): $m \geq 50 \%$.

Após definida a área, foi realizado o corte na imagem, pelo programa IMPIMA (INPE, 1999a). A imagem de satélite apresenta, para cada pixel, um valor de nível de cor cinza (Crosta, 1992). A análise quantitativa de imagens digitais, sobre o comportamento espectral de alvos, deve ser feita com os dados de reflectância devidamente ajustados aos modelos atmosféricos. A seguir, os números digitais são convertidos em valores de reflectância real. Para tanto, a imagem foi processada, foi realizada a correção atmosférica e os valores convertidos em reflectância, segundo Zullo Junior (1994).

Com base no mapa de solos e nas imagens espectrais, foram relacionados os 294 locais de tradagem, porém, quando os pontos de amostragem foram alocados sobre a imagem de satélite, apenas 71 pontos corresponderam a áreas de solo exposto, e esses foram os utilizados na coleta dos dados espectrais pelo programa ENVI (ENVI, 2002). Em cada ponto foi anotada a posição em relação aos eixos x e y. Essa localização foi inserida num banco de dados que extraiu os valores de intensidade de reflectância das seis bandas. A partir desses dados, foram elaborados gráficos espectrais médios, para fins de comparação e caracterização dos solos.
A coleta de dados ocorreu da seguinte forma: 1) os pontos de tradagem e perfis georreferenciados foram sobrepostos às imagens; 2) o cursor foi colocado em tela sobre cada ponto localizado no campo, conhecendo-se o referido solo; 3 ) o pixel foi avaliado quanto ao seu valor espectral, conforme Demattê et al. (2000b) e 4) os pixels que efetivamente representassem o solo foram coletados e utilizados no trabalho.

O objetivo dessa análise foi desenvolver e testar um método para a discriminação do solo, a partir de seus dados espectrais. Para tanto, foi utilizado o procedimento DISCRIM, do programa SAS (SAS, 1999). O método paramétrico avalia o conjunto de dados espectrais de um solo e desenvolve uma equação característica para cada um. Essas equações são testadas pelo próprio programa.

Com o objetivo de verificar a discriminação entre classes de solos, diretamente na imagem de satélite, foi realizada a avaliação da linha do solo; essa técnica relaciona a intensidade de reflectância de um mesmo ponto para as bandas 3 e 4 (Baret et al., 1993).

\section{Resultados e Discussão}

A principal classe de solo mapeada na região é a dos Latossolos, com textura que varia de média-arenosa (150-250 g kg-1 de argila) a muito argilosa (>600 g kg-1 de argila) (Tabela 1), influenciada pelos diferentes materiais de origem da região: o arenito da formação Bauru e o basalto da formação Serra Geral que, em geral, são distróficos ou álicos, e podem ocorrer os eutróficos.

Os Argissolos são, após os Latossolos, os de maior predominância na região. Solos que apresentam perfis com seqüência de horizontes de subsuperfície $\mathrm{Bt} / \mathrm{Bw}$ também são comuns, como é o caso dos Argissolos Latossólicos. Esses solos são originalmente distróficos ou álicos. Os Neossolos Quartzarênicos apresentam teor de argila inferior a $150 \mathrm{~g} \mathrm{~kg}^{-1}$, até $2 \mathrm{~m}$ de profundidade; são bem drenados e ocorrem em relevo de plano a suave-ondulado. As cores variam, de parda às amareladas ou avermelhadas. São encontrados também Nitossolos e Cambissolos de basalto, e estes são eutróficos (Tabela 1).

As variações na forma e intensidade de reflectância são causadas pela interação da energia eletromagnética incidente com os componentes de cada solo (Figura 1). Na banda 1, a intensidade da variação foi menor; todas as classes ficaram dentro de um intervalo de intensidade de reflectância entre 0,0078 , para a classe de 
solo PVA5, e 0,0784, para a classe de solo RQ3 (Figura 1). A maior variação na intensidade de reflectância ocorreu nas bandas 4, 5 e 7 em todas as classes de solo. Entretanto, para os solos RQ3, PVA2 e PVAL5 foi observada maior variação na intensidade de reflectância, a partir da banda 2, em decorrência da textura arenosa, da umidade e de outros fatores característicos desses solos (Tabela 1). Demattê et al. (2000b) também observaram pico de intensidade de reflectância acentuado nos solos com texturas arenosas, em relação aos de texturas argilosas. Na banda 5, todas as curvas atingiram o valor de intensidade de reflectância máximo, em sentido decrescente na direção da banda 7, em virtude da maior absorção dos minerais de argila do solo, nessa faixa de comprimento de onda.

Os LVdf e NV apresentaram-se com intensidade de reflectância mais baixa e tendência de curva horizontal, em relação aos outros solos. Os RQ e PVA5 atingiram intensidades de reflectância bem mais altas (até 0,60 ), com curvas de aspecto ascendente. Tais tendências concordam com as curvas espectrais, obtidas com sensor em laboratório, e coincidem com o padrão do solo original (Epiphanio et al., 1992).

Os solos que possuem classificação textural diferente (Tabela 1) apresentam variações espectrais também diferentes. No geral, os solos mais argilosos apresentam intensidades de reflectância mais baixas, como os LVdf, NV e PVA. Apesar desses solos serem argilosos, diferenças são observadas. Os LVdf e NV têm intensidade de reflectância mais baixa, em virtude do material de origem, e da conseqüente presença de minerais opacos, como a magnetita e ilmenita, que apresentam baixa intensidade de reflectância e promovem a diminuição dessa nos solos (Madeira Netto, 1996). Além disso, os solos mais argilosos retêm mais água do que os arenosos e apresentam maior absorção de energia eletromagnética.

As unidades de mapeamento (UM) LV1 e NV1, por terem teores de argila mais altos, apresentaram curvas espectrais com menores valores de intensidade de reflectância dentre os solos argilosos, o que concorda com Nanni (2000). A unidade de mapeamento PV4-e apresentou menores valores de intensidade de reflectância em todas as bandas do TM; esse comportamento foi melhor definido entre as bandas 4 e 7 . Esse solo apresenta uma baixa intensidade de reflectância, muito semelhante ao $\mathrm{NV}$, e por isso apresentou curva semelhante. A baixa intensidade de reflectância é detectada pelas bandas 5 e 7 do ETM que descrevem, preferencialmente, a absorção relacionada aos grupos

Tabela 1. Valores médios das análises dos solos da região de estudo, profundidade de 0-20 cm.

\begin{tabular}{|c|c|c|c|c|c|c|c|c|c|c|c|c|c|c|c|}
\hline \multirow[t]{2}{*}{ Solo $^{(1)}$} & $\mathrm{pH}$ & MO & $\mathrm{P}$ & $\mathrm{K}$ & $\mathrm{Ca}$ & $\mathrm{Mg}$ & $\mathrm{Al}$ & $\mathrm{H}$ & SB & $\mathrm{T}$ & V & $\mathrm{m}$ & Areia & Silte & Argila \\
\hline & $\mathrm{CaCl}_{2}$ & $\left(\mathrm{~g} \mathrm{dm}^{-3}\right)$ & $\left(\mathrm{mg} \mathrm{dm}^{-3}\right)$ & ---- & & & \multicolumn{4}{|c|}{$\left(\mathrm{mmol}_{\mathrm{c}} \mathrm{kg}^{-1}\right)$--- } & \multicolumn{2}{|c|}{------ (\%)------ } & & & \\
\hline RQo1 & 4,65 & 10,67 & 3,67 & 0,30 & 10,00 & 3,00 & 2,83 & 14,50 & 13,30 & 27,80 & 45,67 & 21,83 & 820,0 & 33,3 & 146,7 \\
\hline RQg3 & 4,38 & 10,33 & 4,00 & 0,38 & 9,17 & 2,83 & 4,50 & 15,17 & 12,38 & 27,55 & 39,00 & 35,67 & 836,7 & 36,7 & 126,7 \\
\hline LV1 & 4,90 & 11,67 & 3,67 & 0,53 & 10,00 & 2,50 & 1,00 & 18,17 & 13,03 & 31,20 & 38,33 & 11,83 & 561,7 & 43,3 & 395,0 \\
\hline LV2 & 4,90 & 12,00 & 3,00 & 0,78 & 8,83 & 5,67 & 2,67 & 13,75 & 15,28 & 29,03 & 46,83 & 23,17 & 674,2 & 56,7 & 269,2 \\
\hline LV3 & 4,25 & 11,89 & 4,06 & 1,31 & 8,17 & 3,11 & 5,44 & 18,94 & 12,59 & 31,53 & 35,83 & 39,86 & 778,3 & 43,3 & 178,3 \\
\hline LVdf1 & 4,99 & 16,00 & 3,17 & 1,02 & 7,54 & 5,21 & 1,71 & 25,04 & 13,77 & 38,81 & 31,08 & 19,38 & 244,0 & 87,3 & 668,7 \\
\hline LVA1 & 4,10 & 14,00 & 3,00 & 0,20 & 1,33 & 1,67 & 10,00 & 34,33 & 3,20 & 37,53 & 8,67 & 75,67 & 690,0 & 33,3 & 276,7 \\
\hline LVA2 & 4,68 & 12,70 & 4,48 & 0,78 & 15,91 & 7,31 & 3,37 & 15,02 & 24,00 & 39,02 & 50,74 & 21,48 & 780,8 & 44,2 & 175,0 \\
\hline PV1 & 4,87 & 13,00 & 3,00 & 0,90 & 10,00 & 2,33 & 0,00 & 13,67 & 13,23 & 26,90 & 48,67 & 0,00 & 726,7 & 33,3 & 240,0 \\
\hline PV2 & 5,23 & 9,33 & 4,67 & 1,67 & 30,67 & 12,67 & 0,00 & 15,00 & 45,00 & 60,00 & 74,67 & 0,00 & 650,0 & 53,3 & 296,7 \\
\hline PV3 & 4,50 & 11,67 & 3,67 & 1,00 & 15,67 & 3,67 & 4,33 & 17,00 & 20,33 & 37,33 & 55,00 & 16,00 & 733,3 & 66,7 & 200,0 \\
\hline PVL & 4,33 & 9,67 & 3,33 & 0,80 & 6,00 & 2,67 & 3,00 & 13,00 & 9,47 & 22,47 & 41,67 & 22,00 & 813,3 & 33,3 & 153,3 \\
\hline PVA1 & 4,53 & 9,33 & 1,33 & 0,60 & 6,67 & 2,33 & 4,00 & 14,33 & 9,60 & 23,93 & 40,00 & 31,67 & 840,0 & 33,3 & 126,7 \\
\hline PVA2 & 4,26 & 10,00 & 4,25 & 0,73 & 4,33 & 1,42 & 7,50 & 23,25 & 6,48 & 29,73 & 24,42 & 52,08 & 793,3 & 48,9 & 157,8 \\
\hline PVA3 & 5,43 & 10,33 & 3,00 & 0,70 & 13,00 & 5,33 & 0,00 & 13,67 & 19,03 & 32,70 & 57,67 & 0,00 & 580,0 & 46,7 & 373,3 \\
\hline PVAL1 & 4,81 & 10,75 & 5,58 & 0,54 & 12,67 & 7,25 & 3,17 & 15,58 & 20,46 & 36,04 & 48,50 & 26,92 & 777,8 & 53,3 & 168,9 \\
\hline PVAL2 & 4,73 & 9,67 & 6,67 & 0,87 & 3,67 & 4,33 & 2,00 & 11,67 & 8,87 & 20,53 & 40,33 & 23,33 & 880,0 & 40,0 & 80,0 \\
\hline NV1 & 5,18 & 17,79 & 4,67 & 1,80 & 31,00 & 11,88 & 1,92 & 22,54 & 44,67 & 67,21 & 61,92 & 9,46 & 236,1 & 96,1 & 667,8 \\
\hline NV2 & 5,40 & 16,33 & 4,00 & 2,07 & 26,33 & 5,00 & 0,00 & 18,00 & 33,40 & 51,40 & 62,67 & 0,00 & 163,3 & 90,0 & 746,7 \\
\hline
\end{tabular}

${ }^{(1)}$ Classes de solos estudadas: RQo - Neossolo Quartzarênico órtico, RQg - Neossolo Quartzarênico hidromórfico, LV1 - Latossolo Vermelho típico argiloso, LV2 - Latossolo Vermelho típico médio-argiloso, LV3 - Latossolo Vermelho típico médio-arenoso, LVdf1 - Latossolo Vermelho distroférrico argiloso, LVA1 - Latossolo Vermelho-Amarelo típico médio-argiloso, LVA2 - Latossolo Vermelho-Amarelo típico médio-arenoso, PV1 - Argissolo Vermelho típico médio-argiloso, PV2 - Argissolo Vermelho abrupto argiloso, PV3 - Argissolo Vermelho abrupto médio-argiloso, PVL - Argissolo Vermelho latossólico médio arenoso, PVA1 - Argissolo Vermelho-Amarelo médio-argioloso, PVA2 - Argissolo Vermelho-Amarelo médio-arenoso, PVA3 - Argissolo Vermelho-Amarelo abrupto argiloso, PVAL1 - Argissolo Vermelho-Amarelo latossólico médio-arenoso, PVAL2 - Argissolo Vermelho-Amarelo latossólico médio-argiloso, NV1 - Nitossolo Vermelho, NV2 - Nitossolo Vermelho e Cambissolos associados. 
$\mathrm{OH}^{-}$e aos minerais de argila, respectivamente. Para as UM com textura mais arenosa (RQ1, RQ3, PVA2, PVAL2), as curvas são mais elevadas (Figura 2). O decréscimo nos teores de ferro, juntamente com o aumento da quantidade de areia, favorece o aumento nos valores da intensidade de reflectância, especialmente próximo à banda 5 do TM-Landsat, o que concorda com Demattê et al. (2000b).
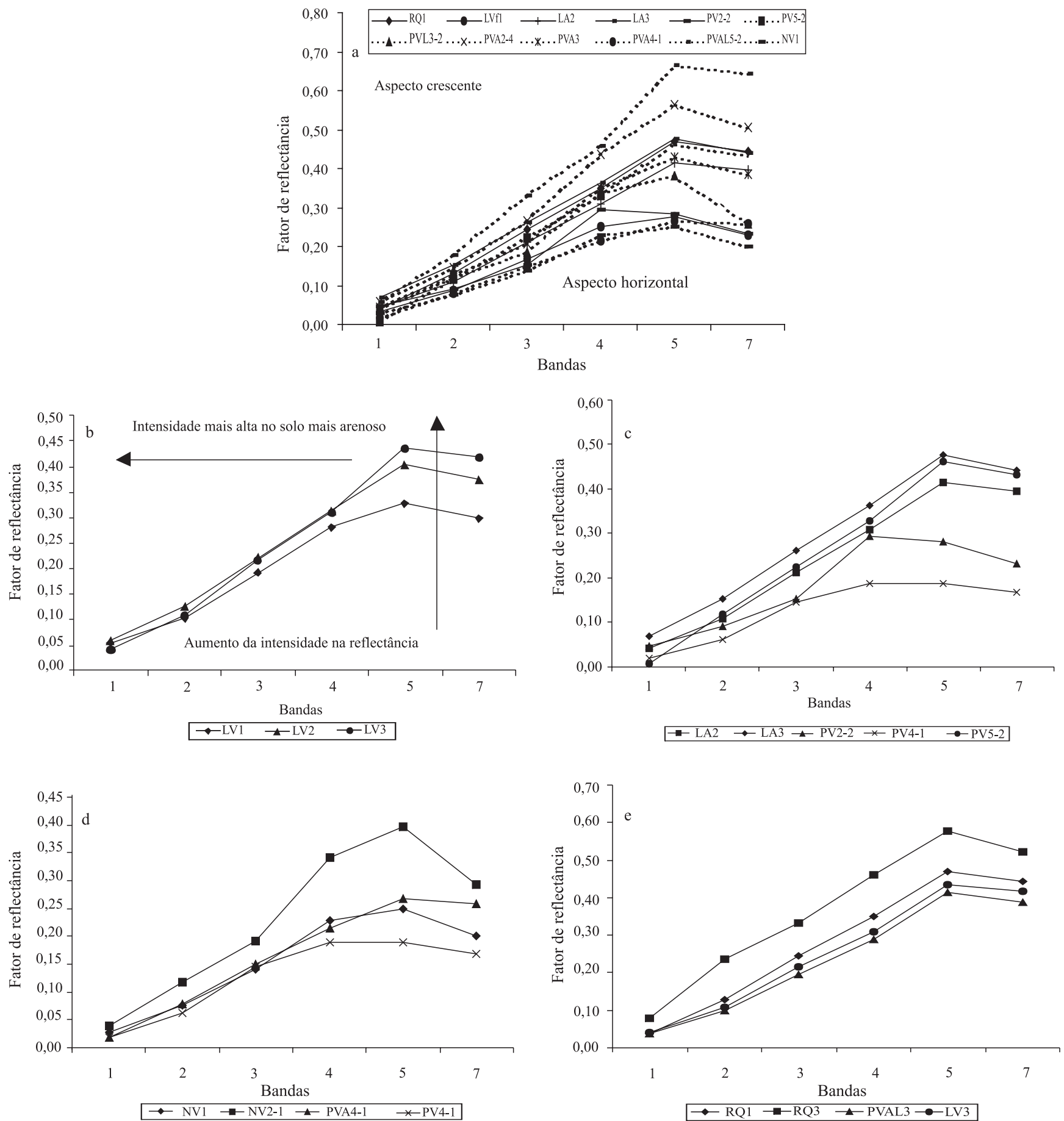

Figura 1. Curvas espectrais médias, determinadas pelo sensor ETM no Landsat: a) curvas dos principais solos da região; b) detalhamento entre Latossolos com diferentes texturas; c), d) e e) comparação entre 4 classes de solos da região. 
O comportamento dos Latossolos foi diferenciado pelas curvas espectrais, apesar de tais solos apresentarem propriedades morfológicas semelhantes (Figura 2). Isso é atribuído ao decréscimo do teor de ferro na camada superficial do solo, na seqüência LVf, LV e LVA, tendo sido observado aumento dos valores de intensidade de reflectância nesta ordem. As cores do horizonte A desses solos variam entre 10R (LVf), 2.5YR (LV) e 5YR (LVA), que apresentam significativa relação com os teores de óxido de ferro (Campos, 2002). Os solos ricos em óxido de ferro - hematita - como o Latossolo Roxo (Latossolo Vermelho férrico) e a Terra Roxa Estruturada (Nitossolo Vermelho eutrófico) apresentam valores de intensidade de reflectância bem menor do que os solos com teores menores de hematita, como os $\mathrm{LV}$ e os PVA. Isto ocorre por causa da absorção de energia promovida pelo óxido, o que pode ser visualizado na ligeira inflexão da linha entre as bandas 3 e 4 (Figura 2), associada à presença desse mineral e a sua in-
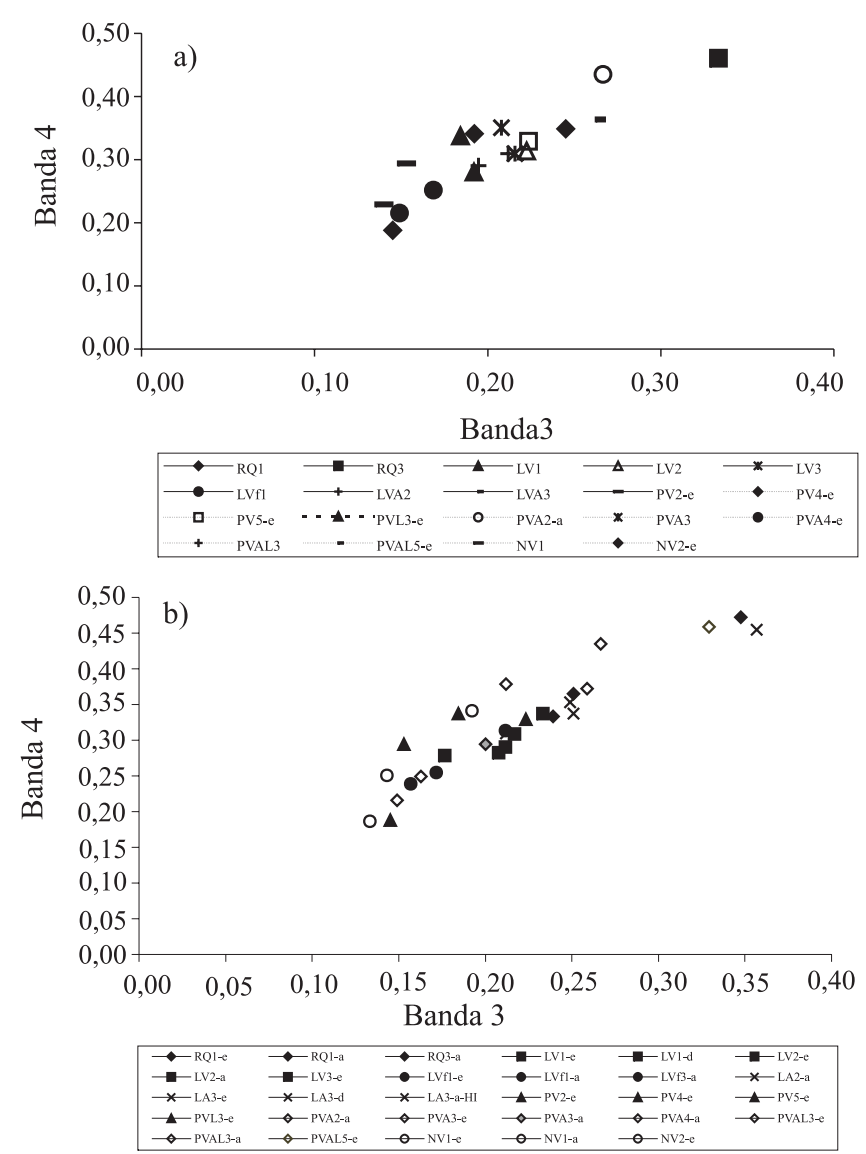

Figura 2. Solos identificados pela textura (a) e pelas propriedades químicas (b). fluência na intensidade de reflectância, conforme verificado por Madeira Netto (1996), em trabalho realizado em laboratório.

A matéria orgânica exerce papel fundamental na absorção de energia analisada pelo espectro eletromagnético (Coleman \& Montgomery, 1987). Os solos mais argilosos como o NV e LVdf, por terem maiores teores de matéria orgânica, apresentaram menor intensidade de reflectância, o que é uma característica desses solos, pois, solos escuros aquecem-se rapidamente durante as horas do dia pela grande absorção da energia radiante (Bowers \& Hanks, 1965; Mathews, 1973). Dentre os Latossolos o LVdf1, por possuir maior teor de matéria orgânica, apresentou menor intensidade de reflectância (Tabela 1).

Os teores mais baixos de matéria orgânica dos solos de textura arenosa, em relação aos de textura argilosa, também contribuíram para a alta intensidade de reflectância que esses solos apresentaram, como ocorrido para a UM PVAL-a (Figura 1).

Foi observado que à medida que o teor de argila diminui, ocorre aumento na intensidade de reflectância e conseqüentemente na forma de curva (Figura 1b). Os Latossolos, divididos em três classes, foram discriminados, o que indica a importância da granulometria na interpretação de um gráfico espectral.

Os solos eutróficos apresentam maior intensidade de reflectância em relação aos solos álicos ou distróficos neste trabalho (Figura 1). Segundo Demattê et al. (1998) isto ocorre porque as bases trocáveis contribuem para a maior refletividade dos solos.

A linha do solo é uma representação sintética da intensidade de reflectância de um número de solos, tomada em duas bandas espectrais. Trata-se de um gráfico de dispersão entre as bandas do visível (3) e infravermelho (4).

A linha de solo foi realizada, considerando-se a textura e a fertilidade dos solos separadamente (Figura 2). Para gerar a relação entre essas bandas, foram utilizados os pontos sobre solo exposto na imagem. Os seguintes fatores indicaram que os pixels amostrados eram basicamente de solos: 1) os pontos encontram-se distribuídos em torno de uma linha a $45^{\circ}$ em relação aos eixos, com baixa dispersão e 2) apresentavam altos valores de $\mathrm{R}^{2}$ (Tabela 2). As equações de tendência de cada solo (Tabela 2) indicam que as assinaturas espectrais, obtidas da imagem, fornecem informações a respeito de solo, não havendo interferências significativas de outros alvos como a vegetação (Baret et al., 1993). 
Cada classe de solo apresenta um padrão espectral que foi transformado em modelo estatístico. Cada classe de solo apresentou variações espectrais diferenciadas ao longo das bandas, podendo ter uma assinatura espectral modelada (Tabela 3).

A análise discriminante para as UM foi feita utilizando-se as bandas do ETM-Landsat B1: (450-520 nm); B2 (520-600 nm); B3 (630-690 nm); B4 (760-990 nm); B5 $(1.550-1.750 \mathrm{~nm})$ e B7 $(2.080-2.350 \mathrm{~nm})$ como as variáveis independentes.

Essas equações podem ser utilizadas, uma vez que se tenham os dados de reflectância obtidos pela conversão dos níveis digitais, de uma cena de certa região onde a cobertura vegetal seja muito baixa ou nula. Os dados de reflectância deverão ser aplicados em todas as equações (Tabela 3). A equação do solo que obtiver o maior valor resultante, terá uma maior probabilidade de ser o solo desconhecido que se deseja identificar. No entanto, esse raciocínio é válido para os solos em regiões de condições semelhantes a do presente trabalho, podendo ser utilizado no auxílio de uma identificação preliminar e rápida.

Entre as amostras analisadas, 80,77\% foram classificadas corretamente e $19,23 \%$ foram confundidas (Tabela 4). Esses valores são próximos aos de Nanni (2000) e inferiores aos de Demattê \& Garcia (1999). A maior confusão foi encontrada para a classe LVA3e, com $75 \%$ de erro, em que as quatro amostras foram classificadas como RQ1-a, LVdf3-a, NVa e PVA3-a, porque as texturas das unidades LVA3, RQ e PVA3 são semelhantes. A granulometria do RQ é inferior a $150 \mathrm{~g} \mathrm{~kg}^{-1}$, o LVA3 de 15\% a 25\%, assim como o PVA3. O erro na classificação é inerente à limitação do sensor em obter informações de amostras de subsuperfície, ou seja, a classificação desse PVA só seria possível com o trabalho em campo.

Tabela 2. Classes de solo, equações e respectivos $\mathrm{R}^{2}$ para a relação entre as bandas 3 e 4 obtidas pela leitura de pixel da cena TM-Landsat.

\begin{tabular}{lcc}
\hline Solo & \multicolumn{1}{c}{ Equação } & $\mathrm{R}^{2}$ \\
\hline RQ e LV & $\mathrm{Y}=1,2618 \mathrm{x}+0,0381$ & 0,96 \\
LVf e LVA & $\mathrm{Y}=1,0787 \mathrm{x}+0,0748$ & 0,98 \\
PVA e NV & $\mathrm{Y}=1,629 \mathrm{x}-0,087$ & 0,67 \\
PVA & $\mathrm{Y}=1,4202 \mathrm{x}+0,0273$ & 0,88 \\
\hline
\end{tabular}

Tabela 3. Equações discriminantes das classes de solos, em função das bandas do ETM-Landsat.

\begin{tabular}{|c|c|}
\hline Classe $^{(1)}$ & Equação discriminante $^{(2)}$ \\
\hline RQ1-e & $\begin{array}{l}-46,4206-228,4600 \mathrm{~B} 1-125,9748 \mathrm{~B} 2-14,8318 \mathrm{~B} 3+199,3307 \mathrm{~B} 4- \\
254,0578 \mathrm{~B} 5+403,4543 \mathrm{~B} 7\end{array}$ \\
\hline RQ1-a & $\begin{array}{l}-62,5108-241,7664 \mathrm{~B} 1-274,8210 \mathrm{~B} 2+39,6622 \mathrm{~B} 3+264,4101 \mathrm{~B} 4- \\
286,0963 \mathrm{~B} 5+437,0335 \mathrm{~B} 7\end{array}$ \\
\hline RQ3-a & $\begin{array}{l}-66,2576-282,5612 \mathrm{~B} 1+303,8616 \mathrm{~B} 2-345,5951 \mathrm{~B} 3+244,5534 \mathrm{~B} 4 \\
-380,9188 \mathrm{~B} 5+584,1653 \mathrm{~B} 7\end{array}$ \\
\hline LV1-e & $\begin{array}{l}-24,8406-37,1991 \mathrm{~B} 1-351,8395 \mathrm{~B} 2+105,4918 \mathrm{~B} 3+314,4517 \mathrm{~B} 4 \\
-173,1839 \mathrm{~B} 5+122,8555 \mathrm{~B} 7\end{array}$ \\
\hline LV1-d & $\begin{array}{l}-30,36133-121,7438 \mathrm{~B} 1-268,5567 \mathrm{~B} 2+114,4559 \mathrm{~B} 3+191,6955 \mathrm{~B} 4 \\
-184,76132 \mathrm{~B} 5+250,3459 \mathrm{~B} 7\end{array}$ \\
\hline LV2-e & $\begin{array}{l}-27,9713-135,1567 \mathrm{~B} 1-156,4192 \mathrm{~B} 2+53,7164 \mathrm{~B} 3+157,4458 \mathrm{~B} 4- \\
168,6005 \mathrm{~B} 5+255,9643 \mathrm{~B} 7\end{array}$ \\
\hline LV2-e & $\begin{array}{l}-38,91776-250,8995 \mathrm{~B} 1-178,9701 \mathrm{~B} 2+43,6185 \mathrm{~B} 3+224,3467 \mathrm{~B} 4- \\
262,2854 \mathrm{~B} 5+357,0606 \mathrm{~B} 7\end{array}$ \\
\hline LV2-a & $\begin{array}{l}-36,9334-44,8428 \mathrm{~B} 1-264,1166 \mathrm{~B} 2+58,4441 \mathrm{~B} 3+181,1672 \mathrm{~B} 4- \\
121,5379 \mathrm{~B} 5+226,8691 \mathrm{~B} 7\end{array}$ \\
\hline LV3-e & $\begin{array}{l}-60,8654-250,7927 \mathrm{~B} 1-54,8743 \mathrm{~B} 2-165,4928 \mathrm{~B} 3+241,5738 \mathrm{~B} 4- \\
325,1146 \mathrm{~B} 5+539,7188 \mathrm{~B} 7\end{array}$ \\
\hline LV3-e & $\begin{array}{l}-48,4788-185,9373 \mathrm{~B} 1-169,4891 \mathrm{~B} 2-7,6112 \mathrm{~B} 3+153,2214 \mathrm{~B} 4- \\
224,6414 \mathrm{~B} 5+416,3678 \mathrm{~B} 7\end{array}$ \\
\hline LV3-a & $\begin{array}{l}-43,0196-201,2953 \mathrm{~B} 1-253,7626 \mathrm{~B} 2+27,9028 \mathrm{~B} 3+293,4075 \mathrm{~B} 4 \\
-292,2899 \mathrm{~B} 5+370,9185 \mathrm{~B} 7\end{array}$ \\
\hline LVdf1-e & $\begin{array}{l}-23,4650-60,7526 \mathrm{~B} 1-464,4936 \mathrm{~B} 2+285,0277 \mathrm{~B} 3+211,8802 \mathrm{~B} 4 \\
-37,1807 \mathrm{~B} 5-11,8664 \mathrm{~B} 7\end{array}$ \\
\hline LVdf1-a & $\begin{array}{l}-23,7360-88,3488 \mathrm{~B} 1-436,8303 \mathrm{~B} 2+226,8088 \mathrm{~B} 3+308,7524 \mathrm{~B} 4 \\
-160,9397 \mathrm{~B} 5+64,7676 \mathrm{~B} 7\end{array}$ \\
\hline LVA2-a & $\begin{array}{l}-43,4150-187,4732 \mathrm{~B} 1-181,3167 \mathrm{~B} 2-25,1457 \mathrm{~B} 3+238,1350 \mathrm{~B} 4 \\
-271,5631 \mathrm{~B} 5+402,0682 \mathrm{~B} 7\end{array}$ \\
\hline LVA3-e & $\begin{array}{l}-42,9682-214,6043 \mathrm{~B} 1+103,8060 \mathrm{~B} 2-155,1885 \mathrm{~B} 3+171,4617 \mathrm{~B} 4 \\
-247,2314 \mathrm{~B} 5+408,5936 \mathrm{~B} 7\end{array}$ \\
\hline LVA3-e & $\begin{array}{l}-48,3079-188,4942 \mathrm{~B} 1-235,5243 \mathrm{~B} 2+33,5837 \mathrm{~B} 3+226,1488 \mathrm{~B} 4 \\
-228,2037 \mathrm{~B} 5+360,9868 \mathrm{~B} 7\end{array}$ \\
\hline LVA3-d & $\begin{array}{l}-64,8797-185,9934 \mathrm{~B} 1-428,5629 \mathrm{~B} 2+199,4147 \mathrm{~B} 3+188,6411 \mathrm{~B} 4 \\
-221,6077 \mathrm{~B} 5+379,5935 \mathrm{~B} 7\end{array}$ \\
\hline LVA3-a-HI & $\begin{array}{l}-49,6555+126,7426 \mathrm{~B} 1-2,4954 \mathrm{~B} 2-29,3977 \mathrm{~B} 3+41,6888 \mathrm{~B} 4- \\
10,1875 \mathrm{~B} 5+148,2586 \mathrm{~B} 7\end{array}$ \\
\hline PVA2-e & $\begin{array}{l}-33,0866-73,4533 \mathrm{~B} 1-291,5132 \mathrm{~B} 2-59,7143 \mathrm{~B} 3+493,2500 \mathrm{~B} 4- \\
307,9804 \mathrm{~B} 5+202,9670 \mathrm{~B} 7\end{array}$ \\
\hline PVA4-e & $\begin{array}{l}-20,5508-74,5665 \mathrm{~B} 1-510,1877 \mathrm{~B} 2+374,8569 \mathrm{~B} 3+203,7012 \mathrm{~B} 4 \\
-84,6764 \mathrm{~B} 5-13,2871 \mathrm{~B} 7\end{array}$ \\
\hline PVA5-e & $\begin{array}{l}-57,4728-316,7338 \mathrm{~B} 1-99,0632 \mathrm{~B} 2-63,1271 \mathrm{~B} 3+221,1016 \mathrm{~B} 4- \\
289,9475 \mathrm{~B} 5+474,0443 \mathrm{~B} 7\end{array}$ \\
\hline PVAL3-e & $\begin{array}{l}-32,3094-7,9050 \mathrm{~B} 1-389,6802 \mathrm{~B} 2+176,0900 \mathrm{~B} 4+212,9716 \mathrm{~B} 4+ \\
152,4540 \mathrm{~B} 5-193,0771 \mathrm{~B} 7\end{array}$ \\
\hline PVA2-a & $\begin{array}{l}-71,5916-199,9395 \mathrm{~B} 1-270,6843 \mathrm{~B} 2-72,9379 \mathrm{~B} 3+369,9924 \mathrm{~B} 4- \\
288,9663 \mathrm{~B} 5+426,5647 \mathrm{~B} 7\end{array}$ \\
\hline PVA3-e & $\begin{array}{l}-56,7563-128,6798 \mathrm{~B} 1-158,5975 \mathrm{~B} 2-252,4119 \mathrm{~B} 3+504,0434 \mathrm{~B} 4 \\
-434,6178 \mathrm{~B} 5+486,5749 \mathrm{~B} 7\end{array}$ \\
\hline PVA3-e & $\begin{array}{l}-46,2815-221,3088 \mathrm{~B} 1-102,0367 \mathrm{~B} 2-190,0473 \mathrm{~B} 3+482,2471 \mathrm{~B} 4 \\
-365,0525 \mathrm{~B} 5+359,1333 \mathrm{~B} 7\end{array}$ \\
\hline PVA3-a & $\begin{array}{l}-48,7805-282,8469 \mathrm{~B} 1+183,4150 \mathrm{~B} 2-288,1546 \mathrm{~B} 3+170,9145 \mathrm{~B} 4 \\
-327,1532 \mathrm{~B} 5+561,8631 \mathrm{~B} 7\end{array}$ \\
\hline PVA4-e & $\begin{array}{l}-22,5322-180,7924 \mathrm{~B} 1-108,3859 \mathrm{~B} 2-36,5654 \mathrm{~B} 3+236,9189 \mathrm{~B} 4 \\
-283,1715 \mathrm{~B} 5+336,0592 \mathrm{~B} 7\end{array}$ \\
\hline PVAL3-e & $\begin{array}{l}-25,4908-238,0719 \mathrm{~B} 1-108,9125 \mathrm{~B} 2-24,5882 \mathrm{~B} 3+260,3924 \mathrm{~B} 4 \\
-288,7759 \mathrm{~B} 5+328,5418 \mathrm{~B} 7\end{array}$ \\
\hline PVAL3-e & $\begin{array}{l}-40,2155-54,3393 \mathrm{~B} 1-210,4351 \mathrm{~B} 2+9,5415 \mathrm{~B} 3+34,6437 \mathrm{~B} 4+ \\
4,0463 \mathrm{~B} 5+222,1358 \mathrm{~B} 7\end{array}$ \\
\hline PVAL3-a & $\begin{array}{l}-67,2498-205,3599 \mathrm{~B} 1-187,6235 \mathrm{~B} 2-37,1543 \mathrm{~B} 3+197,9291 \mathrm{~B} 4 \\
-257,4913 \mathrm{~B} 5+480,1729 \mathrm{~B} 7\end{array}$ \\
\hline PVAL5-e & $\begin{array}{l}-109,4563-331,5272 \mathrm{~B} 1-91,7229 \mathrm{~B} 2-115,9284 \mathrm{~B} 3+217,8660 \mathrm{~B} 4 \\
-371,3010 \mathrm{~B} 5-92,4371 \mathrm{~B} 7\end{array}$ \\
\hline NV1-e & $\begin{array}{l}-20,9665-48,2103 \mathrm{~B} 1-368,8835 \mathrm{~B} 2+135,5378 \mathrm{~B} 3+294,6483 \mathrm{~B} 4 \\
-92,4371 \mathrm{~B} 5+19,2430 \mathrm{~B} 7\end{array}$ \\
\hline NV1-e & $\begin{array}{l}-21,2387-67,4617 \mathrm{~B} 1-322,8258 \mathrm{~B} 2+103,1875 \mathrm{~B} 3+233,3927 \mathrm{~B} 4 \\
-30,3284 \mathrm{~B} 5+18,7911 \mathrm{~B} 7\end{array}$ \\
\hline NV1-a & $\begin{array}{l}-13,4694-71,7484 \mathrm{~B} 1-331,4813 \mathrm{~B} 2+217,0301 \mathrm{~B} 3+155,1439 \mathrm{~B} 4 \\
-61,7458 \mathrm{~B} 5+31,1940 \mathrm{~B} 7\end{array}$ \\
\hline NV2-e & $\begin{array}{l}-33,0766-50,0515 \mathrm{~B} 1-385,3550 \mathrm{~B} 2-143,9981 \mathrm{~B} 3+294,7166 \mathrm{~B} 4 \\
+48,4649 \mathrm{~B} 5-63,3923 \mathrm{~B} 7\end{array}$ \\
\hline
\end{tabular}

(1)Classes de solos (a: álico, $\mathrm{m} \geq 50 \%$; d: distrófico, $\mathrm{V}<50 \%$; e: eutrófico, $\mathrm{V} \geq 50 \%)$. (2)Equações discriminantes para cada classe de solo da área de estudo: B1: 450-520 nm; B2: 520-600 nm; B3: 630-690 nm; B4: 760-990 nm; B5: $1.550-1.750 \mathrm{~nm}$ e B7: $2.080-2.350 \mathrm{~nm}$. 
Verifica-se que, em muitos casos, os Latossolos são discriminados dos Argissolos pelas propriedades dos horizontes subsuperficiais Bw e Bt (Tabela 4). Um solo com Bt, por exemplo, pode reter mais água que um com Bw. Um caso característico é o dos Argissolos abruptos, que retêm mais água que um Argissolo típico (Tabela 4).

Demattê \& Garcia (1999) testaram as equações na caracterização de solos, com os dados analíticos de reflectância espectral, e obtiveram uma eficiência de $100 \%$ nos solos estudados, exceto o LVdf e o NV, que tinham apenas uma amostra utilizada na classificação.

Tabela 4. Análise discriminante, não-paramétrica, dos grupos de solos, número de observações, classificações e erro para os dados obtidos do sensor orbital.

\begin{tabular}{|c|c|c|c|}
\hline Solos $^{(1)}$ & $\begin{array}{c}\text { Corretas }^{(2)} \\
(\%)\end{array}$ & $\begin{array}{c}\text { Erradas }^{(3)} \\
(\%)\end{array}$ & $\begin{array}{c}\text { Solos } \\
\text { confundidos }^{(4)}\end{array}$ \\
\hline RQo-e & 100 & 0 & - \\
\hline RQo-a & 100 & 0 & - \\
\hline RQg-a & 100 & 0 & - \\
\hline LV1-a & 100 & 0 & - \\
\hline LV1-d & 100 & 0 & - \\
\hline LV2-e & 100 & 0 & - \\
\hline LV2-e & 100 & 0 & - \\
\hline LV2-d & 50 & 50 & LVf3-a \\
\hline LV3-e & 100 & 0 & - \\
\hline LV3-e & 80 & 20 & PVAL3-a \\
\hline LV3-d & 100 & 0 & - \\
\hline LVdf-e & 50 & 50 & LVf1-eNV1-e \\
\hline LVdf-a & 100 & 0 & - \\
\hline LVA1-a & 100 & 0 & - \\
\hline LVA2-e & 50 & 50 & PVA3-a \\
\hline LVA3-e & 25 & 75 & RQ1-a, LVdf3-a, PVA3-a \\
\hline LVA2-d & 100 & 0 & - \\
\hline LVA3-a-HI & 100 & 0 & - \\
\hline PVA1-e & 100 & 0 & - \\
\hline PVA2-e & 100 & 0 & - \\
\hline PVA3-e & 100 & 0 & - \\
\hline PVAL-e & 100 & 0 & - \\
\hline PVA1-a & 100 & 0 & - \\
\hline PVA2-e & 100 & 0 & - \\
\hline PVA2-e & 100 & 0 & - \\
\hline PVA2-a & 100 & 0 & - \\
\hline PVA3-e & 100 & 0 & - \\
\hline PVAL1-e & 100 & 0 & - \\
\hline PVAL1-e & 100 & 0 & - \\
\hline PVAL1-a & 100 & 0 & - \\
\hline PVAL2-e & 100 & 0 & - \\
\hline NV1-e & 33 & 67 & PV4-e, NV2-e \\
\hline NV1-e & 100 & 0 & - \\
\hline NV1-a & 100 & 0 & - \\
\hline NV2-a & 100 & 0 & - \\
\hline Total & 80,77 & 19,23 & \\
\hline
\end{tabular}

(1)Classes de solo (a: álico, $\mathrm{m} \geq 50 \%$; d: distrófico, $\mathrm{V}<50 \%$; e: eutrófico, $\mathrm{V} \geq 50 \%)$. ${ }^{(2)}$ Número de amostras e porcentagem classificada corretamente pela equação discriminante para cada classe de solo. ${ }^{(3)}$ Número de amostras e porcentagem classificada erroneamente pela equação discriminante para cada classe de solo. ${ }^{(4)}$ Classes de solos que foram confundidas no processo de classificação pelas equações discriminantes.
Nos trabalhos de Nanni (2000) foram discriminados solos pela análise estatística discriminante, utilizando-se informações obtidas nos níveis de laboratório e orbital, que permitiram mais de $70 \%$ e $80 \%$ de acertos no nível orbital e laboratorial, respectivamente.

Nos LVdf2-a e LVdf3-a houve um erro de 50\%. Esses solos podem ser confundidos por apresentarem as mesmas propriedades químicas, e semelhanças nas propriedades físicas (textura média argilosa com $25 \%$ a $35 \%$, e $15 \%$ a $25 \%$ de argila, respectivamente). O LVdf3-e e o PVAL3-a foram confundidos em $20 \%$ das amostras, por apresentarem semelhanças nas propriedades químicas e físicas. O LVdf1-e foi confundido, em 50\% das amostras, com o LVdf1-e e NV1-e, que se diferem nos horizontes em razão do teor de ferro.

O LVA2-e foi confundido em 50\% com os PVA3-a, por serem semelhantes em suas propriedades físicas superficiais, onde se diferem por apresentar Bt no PVA, com acúmulo da argila no horizonte subsuperficial. O LVA3-e foi confundido, em 75\%, com o RQ1-a, LVf3-a PVA3-a, em razão da semelhança nas características superficiais, como o LVdf1-a e PVA3-a; no caso do RQ1-a, que apresenta maior teor de argila que LVA3-e, por ser um Neosssolo Quartzarênico, pode ter reflectância semelhante. O NV1-e foi confundido, em 67\%, com o PV4-e e NV2-e, em razão das propriedades químicas semelhantes, e pelo número de amostras de PV4-e e NV2-e (apenas uma).

Em relação às respectivas bandas estudadas, 5 e 7 foram as que melhor discriminaram o solo, na comparação das médias (Tabela 5).

Tabela 5. Teste de comparação de médias dos solos para as respectivas bandas $^{(1)}$.

\begin{tabular}{lcccccc}
\hline Solos & $\mathrm{B}^{(2)}$ & $\mathrm{B}^{(2)}$ & $\mathrm{B}^{(2)}$ & $\mathrm{B}^{(2)}$ & $\mathrm{B}^{(2)}$ & $\mathrm{B}^{(2)}$ \\
\hline RQ & $0,0588 \mathrm{a}$ & $0,1833 \mathrm{a}$ & $0,2892 \mathrm{a}$ & $0,4049 \mathrm{a}$ & $0,5235 \mathrm{a}$ & $0,4824 \mathrm{a}$ \\
LV & $0,0485 \mathrm{a}$ & $0,1126 \mathrm{bc}$ & $0,2143 \mathrm{~b}$ & $0,3067 \mathrm{a}$ & $0,4109 \mathrm{a}$ & $0,3882 \mathrm{a}$ \\
LVf & $0,0322 \mathrm{a}$ & $0,0855 \mathrm{bc}$ & $0,1686 \mathrm{~b}$ & $0,2518 \mathrm{a}$ & $0,2761 \mathrm{~b}$ & $0,2306 \mathrm{~b}$ \\
LA & $0,0658 \mathrm{a}$ & $0,1490 \mathrm{ab}$ & $0,2571 \mathrm{a}$ & $0,3577 \mathrm{a}$ & $0,4701 \mathrm{a}$ & $0,4366 \mathrm{a}$ \\
PVA & $0,0284 \mathrm{a}$ & $0,0980 \mathrm{bc}$ & $0,1765 \mathrm{~b}$ & $0,2873 \mathrm{a}$ & $0,3284 \mathrm{~b}$ & $0,2725 \mathrm{~b}$ \\
PVA & $0,0423 \mathrm{a}$ & $0,1197 \mathrm{bc}$ & $0,2181 \mathrm{~b}$ & $0,3364 \mathrm{a}$ & $0,4500 \mathrm{a}$ & $0,4176 \mathrm{a}$ \\
NV & $0,0291 \mathrm{a}$ & $0,0812 \mathrm{c}$ & $0,1474 \mathrm{~b}$ & $0,2454 \mathrm{a}$ & $0,2706 \mathrm{~b}$ & $0,2146 \mathrm{~b}$ \\
\hline CV $(\%)$ & 64,00 & 32,44 & 21,23 & 19,64 & 20,86 & 21,10 \\
\hline
\end{tabular}

(1)Médias seguidas de mesma letra, na coluna, não diferem entre si a 5\% de probabilidade, pelo teste de Tukey. ${ }^{(2)} \mathrm{B} 1:$ 450-520 nm; B2: 520$600 \mathrm{~nm}$; B3: 630-690 nm; B4: 760-990 nm; B5: 1.550-1.750 nm e B7: $2.080-2.350 \mathrm{~nm}$. 


\section{Conclusões}

1. É possível caracterizar e discriminar, espectralmente, por imagens de satélites, os solos na região estudada.

2. A granulometria e a matéria orgânica são os principais atributos atuantes na reflectância orbital.

3. Solos mais argilosos refletem menos que os mais arenosos, ao nível de sensor, no satélite.

4. Solos eutróficos refletem mais que solos álicos e podem ser discriminados superficialmente.

5. A coleta de dados, por imagem de satélite, é eficiente na identificação de solos da região.

6. A interpretação desse tipo de gráfico de dispersão permite diagnosticar, com maior segurança, se o pixel coletado na imagem refere-se a solos ou à vegetação.

\section{Referências}

BARET, F.; JACQUEMOUD, S.; HANOCQ, J.F. The soil line concept in remote sensing. Remote Sensing of Environment, v.7, p.11-18, 1993.

BEN-DOR, E. Quantitative remote sensing of soil properties. Advances in Agronomy, v.75, p.173-243, 2002.

BOWERS, S.A.; HANKS, R.J. Reflection of radiant energy from soils. Soil Science, v.100, p.130-137, 1965.

CAMARGO, M.N.; KLANT, E.; KAUFFMAN, J.H. Classificação de solos usada em levantamentos pedológicos no Brasil. Boletim Informativo da Sociedade Brasileira de Ciência do Solo, v.12, p.11-13, 1987.

CAMPOS, R.C. Determinação da cor dos solos e sua utilização na estimativa dos teores de hematita. 2002. 59p. Dissertação (Mestrado) - Escola Superior de Agricultura “Luiz de Queiroz”, Piracicaba.

COLEMAN, T.L.; MONTGOMERY, O.L. Soil moisture, organic matter and iron content effect on the spectral characteristics of selected Vertisols and Alfisols in Alabama. Photogrametric Engineering and Remote Sensing, v.53, p.1659-1663, 1987.

CROSTA, A.P. Processamento digital de imagens de sensoriamento remoto. Campinas: UNICAMP, 1992. 170p.

DEMATTÊ, J.A.M.; CAMPOS, R.C.; ALVES, M.C. Avaliação espectral de solos desenvolvidos em uma toposseqüência de diabásio e folhelho da região de Piracicaba, SP. Pesquisa Agropecuária Brasileira, v.35, p.2447-2460, 2000a.

DEMATTÊ, J.A.M.; GARCIA, G.J. Alteration of soil properties through a weathering sequence as evaluated by spectral reflectance. Soil Science Society of America Journal, v.63, p.327-342, 1999.
DEMATTÊ, J.A.M.; GARCIA, G.J.; PROCHNOW, L.I. Variações induzidas de atributos químicos e sua influência na intensidade de reflectância espectral de três solos do Estado do Paraná. Revista Brasileira de Ciência do Solo, v.22, p.479-490, 1998.

DEMATTÊ, J.A.M.; HUETE, A.R.; FERREIRA, L.G.; ALVES, M.C.; NANNI, M.R.; CERRI, C.E. Evaluation of tropical soils through ground and orbital sensors. In: INTERNATIONAL CONFERENCE ON GEOESPATIAL INFORMATION IN AGRICULTURE AND FORESTRY, 2 ${ }^{\text {nd }}, 10-12$ Jan, 2000, Lake Buena Vista, Florida. Proceedings. Florida, 2000b.

EMBRAPA. Centro Nacional de Pesquisa de Solos (Rio de Janeiro, RJ). Normas e critérios para levantamentos pedológicos. Rio de Janeiro, 1996. 94p.

EMBRAPA. Centro Nacional de Pesquisa de Solos (Rio de Janeiro, RJ). Sistema brasileiro de classificação de solos. Brasília: SPI, 1999. 412p.

ENVI. Environment for Visualizing Images. Guia do ENVI 3.5 em Português. Disponível em: <http://www.envi.com.br>. Acesso em: 2002.

EPIPHANIO, J.C.N.; FORMAGGIO, A.R.; VALVFRIANO, M.; OLIVEIRA, J.B. Comportamento espectral de solos do Estado de São Paulo. São José dos Campos: INPE, 1992. 131p.

IBGE. Estatística de produção mundial. Disponível em: <http:// www.agricultura.gov.br/sarc/dfPVA/prodecer.htm>. Acesso em: out. 2003a.

IBGE. Levantamento sistemático de produção agrícola. Disponível em: <http://www.ibge.gov.br/home/estatistica/ indicadores/agropecuaria/lspa/default.shtm>. Acesso em: out. 2003b.

INPE. Software Spring: versão 3.6. Disponível em: <www.inpe.br>. Acesso em: 1999.

INSTITUTO DE GEOCIÊNCIA E CIÊNCIAS EXATAS. Mapa geológico do Estado de São Paulo: folha Marília SF-22-Z-A, escala 1:250.000. Rio Claro, 1982.

LEMOS, R.C. de; SANTOS, R.D. dos. Manual de descrição e coleta de solo no campo. 3.ed. Campinas: Sociedade Brasileira de Ciência do Solo, 1996. 84p.

LILLESAND, T.M.; KIEFER, R.W. Remote sensing and image interpretation. New York: John Wiley \& Sons, 2000. 724p.

MADEIRA NETTO, J.S. Spectral reflectance properties of soils. Photo Interpretation, v.34, p.59-70, 1996.

MATHEWS, H.L. Spectral reflectance of selected Pennsylvania soils. Soil Science Society of America Proceedings, v.37, p.421-424, 1973.

McBRATNEY, A.B.; MENDONÇA SANTOS, M.L.; MINASNY, B. On digital soil mapping. Geoderma, v.117, p.3-52, 2003.

NANNI, M.R. Dados radiométricos obtidos em laboratório e no nível orbital na caracterização e mapeamento dos solos. 2000. 
366p. Tese (Doutorado) - Escola Superior de Agricultura "Luiz de Queiroz”, Piracicaba.

OLIVEIRA, J.B. Pedologia aplicada. Campinas: Funep, 2001. 414p.

RAIJ, B. van; QUAGGIO, J.A.; CANTARELLA, H.; FERREIRA, M.E.; LOPES, A.S.; BATAGLIA, C.O. Análise química do solo para fins de fertilidade. Campinas: Fundação Cargil, 1987. 170p.

SAS INSTITUTE (Cary, Estados Unidos). SAS/STAT user's guide: version 8.2. Cary, 1999. 291p.
SHEPHERD, K.D.; WALSH, M.G. Development of reflectance spectral libraries for characterization of soil properties. Soil Science Society of America Journal, v.66, p.988-998, 2002.

VITORELLO, I.; GALVÃO, L.S. Spectral reflectance characterization of tropical soil types. São José dos Campos: Inpe, 1999. p.342-349.

ZULLO JUNIOR, J. Correção atmosférica de imagens de satélite e aplicações. 1994. 191p. Tese (Doutorado) - Universidade de Campinas, Campinas.

Recebido em 2 de março de 2004 e aprovado em 4 de maio de 2005 